\title{
Supplementing a training procedure with the use of a cadaver
}

It's hard enough for an IACUC to cope with problems directly related to the use of animals in research, education and product testing, but when an IACUC gets drawn into controversies that are tangential to its mission, its problems can quickly escalate. The Great Eastern University IACUC approved Dr. Sam Messinger's use of pigs for training surgeons in natural orifice translumenal endoscopic surgery (NOTES) and specifically for removing the gall bladder through the mouth of the patient. In this procedure, the endoscope and associated instruments are inserted in the patient's mouth and down into the stomach. The surgeon then makes small incisions through the wall of the stomach and eventually removes the gall bladder through the patient's mouth. Messinger was the only surgeon at Great Eastern trained in the technique, and he was anxious to help his colleagues learn the procedure. The IACUC training protocol proposed an acute study in which the pigs would be anesthetized during the procedure then euthanized without awakening.

Before beginning the study, Messinger sent an amendment to the IACUC. With the concurrence of the Department of Anatomy, he proposed to have a human cadaver in the same room with the pigs to enable the surgeons to visualize concurrently the gross anatomical differences between humans and pigs in the areas of interest. The IACUC office did not know whether handling such an amendment was within its authority, so the office staff asked the IACUC chairman. The chairman was unsure, so he asked the Attending Veterinarian (AV) for an opinion. The AV said he had no inherent objection but suggested sending the request to the Institutional Review Board (IRB, the oversight body for human research studies that is analogous to the IACUC). The IRB was equally unsure, so it asked the Institutional Biosafety Committee (IBC) for an opinion, and the IBC said it was an IACUC issue. The IACUC then asked the Institutional Official (IO) to become involved. The IO asked the Dean for her input, and the Dean quickly arranged a meeting with herself, the AV, and the IACUC, IRB and IBC chairpersons.

Do you think that Messinger's amendment is reasonable for the proposed training? What position would you take if you were part of the Dean's meeting?

\section{RESPONSE}

\section{Passing the buck}

Donna L. Goldsteen, BS, RLATG, CMAR, CPIA Shameen Afif-Rider, BS, RLATG, CPIA, RAC \& Youngmi Girard, BSRN, COHN-S, MBA

Passing the buck on an issue that one doesn't know how or want to deal with is unfortunately quite common. We elected to address this scenario by examining each committee's responsibility.

The Institutional Review Board (IRB) is responsible for determining whether the research is in accordance with the Health and Human Services Policy for Protection of Human Research Subjects ${ }^{1}$. In that policy, a human subject is defined as "a living individual." Thus, IRB approval should have been obtained before the body was made available for use at this medical school. Hence, the IRB has no need for further action in this case.
The Institutional Biosafety Committee (IBC) is responsible for determining whether the procedure is in accordance with the institution's biosafety program and other external requirements and guidelines to assure safe practices in handling tissues, viruses and bacteria. Personnel handling the cadaver or animals would be required to attend general occupational health and safety training in order to carry out this type of work. Unless clinical information about the cadaver indicates that there is a potential infectious exposure risk, the IBC has no need for further action in this case.

According to the Public Health Service Policy on Humane Care and Use of Laboratory Animals, "the IACUC shall conduct a review of those components related to the care and use of animals and determine that the proposed research projects are in accordance with this Policy" The Animal Welfare Act Regulations state that a proposal to conduct an activity involving animals must contain statements identifying the species and numbers used; the rationale for involving animals; a description of the proposed use of animals, including final disposition of the animal; and descriptions of methods to alleviate or decrease pain and distress and methods of euthanasia ${ }^{3}$. Animal well-being should be the primary focus of the IACUC. Most facilities try to separate animals from potentially distressful situations; for example, necropsies are not usually done in rooms that house live animals. To decrease potential distress to the pigs caused by the presence of a cadaver, the IACUC should recommend that the pigs be anesthetized sufficiently before the cadaver is brought into the room.

The Attending Veterinarian (AV) is responsible for the surgical procedure done on the pigs. The AV needs to assure that the cadaver bag is transferred to the facility in a manner that prevents any potential contamination of the animals, 